Original Research Paper

\title{
Study of Properties of a Dispersant Used for Extracting Oil with High Gas-Oil Ratio
}

\author{
Vladimir Verbitsky, Aleksey Dengaev, Oleg Zubkov and Dmitry Koshkin \\ Gubkin Russian State University of Oil and Gas, Moscow, Russia
}

\author{
Article history \\ Received: 07-11-2015 \\ Revised: 19-02-2016 \\ Accepted: 23-02-2016 \\ Corresponding Author: \\ Vladimir Verbitsky, \\ Gubkin Russian State \\ University of Oil and Gas, \\ Moscow, Russia \\ Email: vsverbitsky@gmail.com
}

\begin{abstract}
The article presents the bench test results for a dispersant manufactured by Izhnefteplast Company, which specializes in batch production of unique submersible pumping equipment for oil extraction. The submersible equipment is unique in that the working elements of the pumping equipment are made of polymeric materials unlike the conventional concept of pumping machinery for oil production. The relevance of the bench tests is determined by the fact that submersible pumping equipment faces the problem of pumping out well products containing a good deal of free gas in actual field conditions. Gubkin Russian State University of Oil and Gas has provided background to carry out bench tests of submersible pump equipment operating on model gasfluid mixtures at various input conditions (pressure, temperature, electric motor shaft speed, feed, etc.). The bench testing is aimed at development of a procedure for dispersing efficiency determination in pre-start operation modes of submersible electrical centrifugal pumps for oil extraction.
\end{abstract}

Keywords: Well, Well Products, Gas-Fluid Mixture, Free Gas, Dispersing Steps, Electrical Centrifugal Pump, Research Methodology, Test Bench

\section{Introduction}

The essential share of oil extraction in Russia is provided by a mechanized method using submersible electrical centrifugal pumping units (ECP units). As of January 2015, the oil was extracted from 91,798 Russian wells equipped with ECP units (Statistics, 2015).

In most cases, free gas is released from oil causing substantial complications during pressure drawdown: Head and rate characteristics decrease when gas content is high at outlets of submersible pumping systems. Occasions when "pump starvation" of a submersible pumping unit occurs are not rare.

There are a number of methods for prevention of the adverse effect of free gas at the intake of submersible pumping systems, which can be divided into two groups:

- Increase of intake pressure of a submersible electrical centrifugal pump

- Separation and/or dispersion of free gas at the intake of a submersible electrical centrifugal pump

Using the methods of the first group to prevent adverse effect of free gas on operation of ECP units is currently impracticable due to a whole number of reasons, including reduction of drawdown and well discharge.

At the present time, submersible gas separators of electrical centrifugal pumps have gained wide application in the oil industry. Using gas separators as a part of submersible electrical centrifugal units allows operating a well with high content of free gas (up to $80 \%$ vol.). Stable operation of a submersible pumping unit is ensured by high separation capacity of gas separators, but, at the same time, energy characteristics of operation of a gas-liquid lift represented by pump and compressor tubes degrade. In some cases, the flowing mode induced by the separation effect can start in the annular space of the well. But more serious problems are the emergency modes, which can occur due to non-optimal operation of gas separators when pumping gas-fluid mixtures with high content of weighted particles (mechanical impurities) (Dengayev et al., 2005).

Analysis of ECP unit operation in wells of some oil refining enterprises in Russia allowed determining criteria of unjustified application of gas separators: Average value of free gas at the intake of ECP units with gas separators in the category less than $5 \%$ falls on the well stock- $73 \%$ of the total number of the wells under 
consideration (Fig. 1). In the researched wells (approximately 3,000 of the production wells of Western Siberia), power costs of $4,200 \mathrm{~kW}^{*}$ hour can be decreased by excluding gas separators from the ECP units.

Increased attention is paid to the matter of power efficiency in the world today. Up to $70 \%$ of the total power costs of the oil company (Fig. 1) is used for production of liquid hydrocarbons by mechanical methods for well product lifting. This is why increased attention should be paid to a justified method for well operation and selection of certain equipment.

In wells with inconsiderable content of gas (less than $30 \%$ ), dispersing devices or multi-phase pumps can be used as a gas-stabilizing module for gas-fluid mixture preparation. Sufficient quantity of such devices is produced in batches now, however the principles of selection and efficiency modes of their operation have not been determined, which makes this research relevant from the scientific point of view.

\section{Theoretical Background and Literature Review}

The research was carried out on the test bench, the diagram of which is shown in Fig. 2. Similar to the findings of research provided in (Drozdov, 1982), "waterSAS-air" combination (SAS, superficially-active substance-disolvan 4411, concentration- $0.05 \%$ ) was accepted as the model mixture. In the course of the experiments, dispersive ability of gas bubbles at the outlet was maintained at a level of $150-200 \mathrm{mcm}$ according to the recommendations set forth in (Vasiliev et al., 1961).

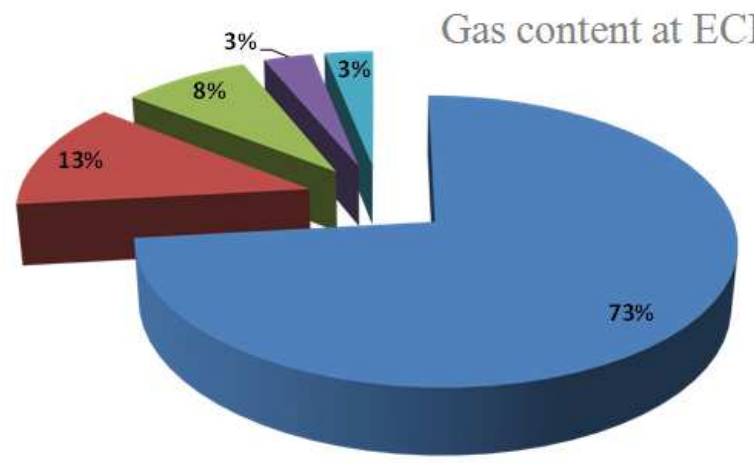

- Below $5 \%$

- From 5 to $10 \%$

= From 10 to $20 \%$

- From 20 to $30 \%$

- Above $30 \%$

Fig. 1.Distribution of the active well stock equipped with ECP by the criterion: "Intake gas content" (oil company of Western Siberia)

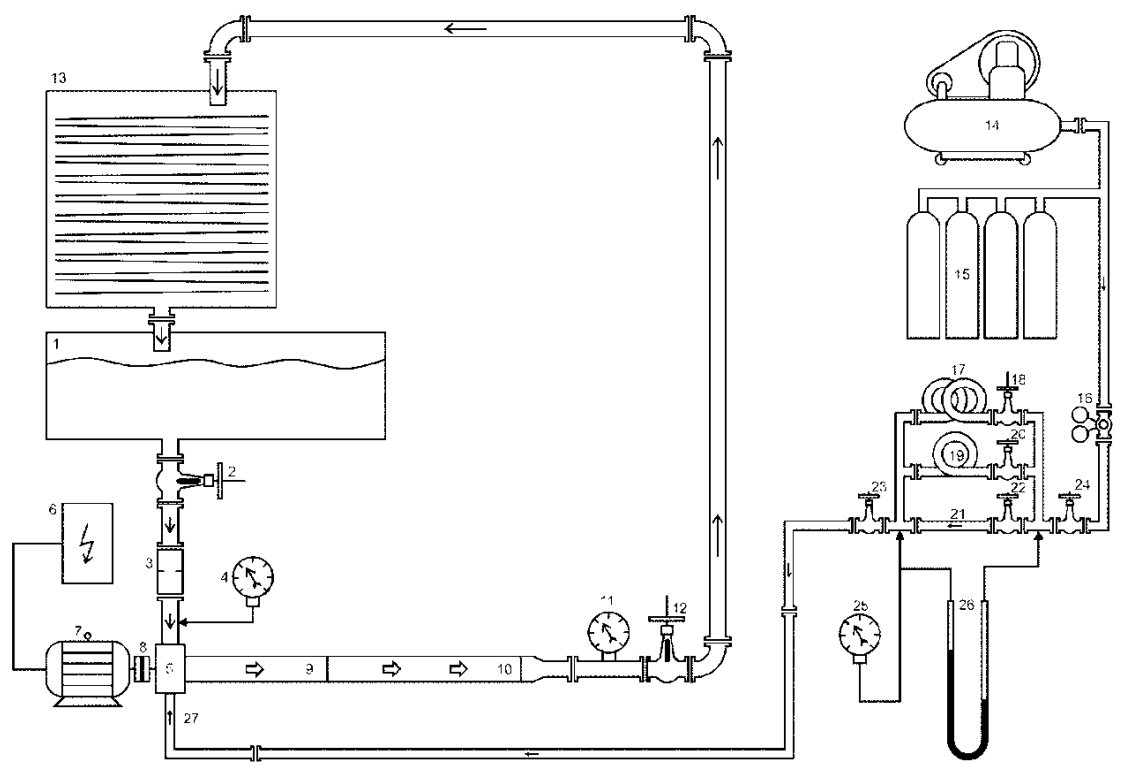

Fig. 2. Diagram of the bench for testing the multi-phase pump on a model coarsely dispersed gas-fluid mixture: 1- working fluid tank; 2- shut-off valve; 3- flow meter; 4, 11, 25- pressure sensors; 5- mixer; 6- variable frequency drive; 7- electric motor; 8torque coupler; 9- researched pump with DS-100 dispersing steps; 10- VNN-79 vortex electrical centrifugal pump; 12, 18, 20, 22, 23, 24- control valves; 13- gravitational separator; 14- compressor; 15- the receiver system cylinders; 16- reducer; 17, 19, 21- air tubes of various resistance degrees; 26- differential pressure gauge 


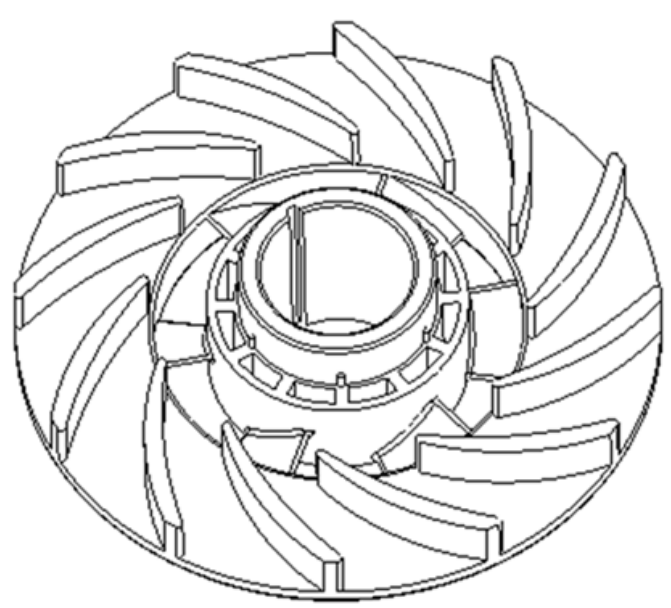

(a)

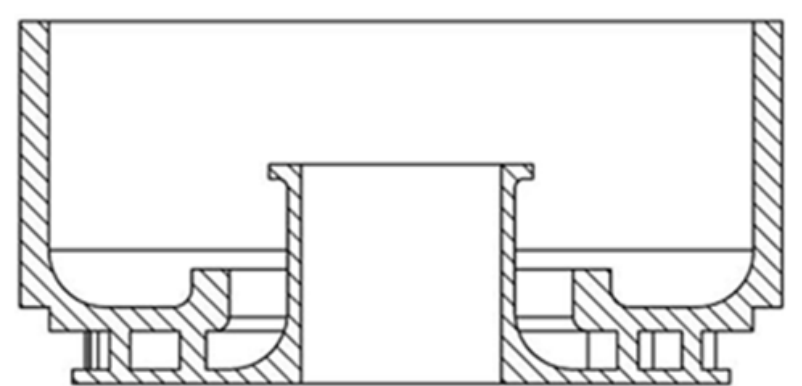

(b)

Fig. 3. Principle diagram of elements of a DS-100 dispersing step: Working wheel (a) and guiding device (b)

Constant excess pressure of $0.1 \mathrm{MPa}$ was maintained at the researched pump intake, air supplied by a compressor entered into the mixer where the gasfluid mixture formed. Volumetric flow rate of the fluid was measured with an ultrasonic flow meter. Airflow was monitored and controlled using a rheometric bench and a mounted differential pressure gauge with calibrated diaphragm.

The research was conducted with discrete adjustment of gas supply to the pump inlet; in particular, gas was supplied from minimum to maximum values of volumetric consumption gas content up to the pump starvation. The bench diagram and the research procedure are provided in detail in (Drozdov, 1982; Dengaev, 2005; Drozdov et al., 2011).

The object of the research is DS-100 dispersing steps (72 stages with nominal supply of $100 \mathrm{~m}^{3} / \mathrm{day}$ ). The dispersing steps are shown as semi-open wheels made of a polymeric material (Fig. 3a) and guiding devices with increased flow cross-section in channels for gas-fluid mixture pumping (material-ChN16D6GH2 heat-resistant high-alloy cast iron, Fig. 3b).

\section{Materials and Methods}

The procedure for bench tests of submersible electrical centrifugal pump on the model gas-fluid mixture consists of two stages: 1. Determination of head and power characteristics using water as a medium to perform comparative analysis of the characteristics versus the pump nameplate data; 2. Determination of degradation degree of head and flow rate characteristics when free gas is supplied as a part the model gas-fluid mixture.

The installation diagram of the test bench (Fig. 2) looks as follows: Electric motor- 7, torque coupler- 8, mixer- 5 , the researched pump with dispersing steps- 9 and electrical centrifugal pump of vortex type- 10 are installed on specially designed supports in succession. The researched pumping equipment is connected to the electric motor- 7 using alignment mechanisms following the position of shaft alignment. Power is supplied to the electric motor using an actuator integrated with the variable frequency drive- 6 . The variable frequency drive- 6 is designed for rated current supplied to the electric motor- 7. Torque from the electric motor- 7 is supplied with the torque coupler- 8 , which is connected with the shaft of the researched equipment- 9,10 . The mixer- 5 is a chamber with a filter to support the dispersion setting at the intake of the researched equipment- 9,10 . The mixer- 5 is connected on one side with the torque coupler- 8 and on the other side-with inlet of the researched pump with dispersing steps- 9. In the upper part of the mixer- 5 there is a connection pipe with a fluid flow meter- 3 and in the lower part- the air supply pipeline- 27 . Gate valve- 2 is provided at the intake of the researched equipment- 9,10 for fluid feed adjustment. To adjust air supply at the intake of the researched equipment- 9,10 , there is a rheometric bench consisting of: Reciprocating compressor- 14, gas cylinders- 15 , reducer- 16 , control valves- $18,20,22,24$, air tubes with various resistance degrees- 17, 19, 21, 23, differential pressure gauge- 26 and pressure sensor- 25 . When using the rheometric bench, air can be supplied to the inlet of the researched equipment- 9, 10 with overpressure and volumetric airflow rate can be measured in the process.

The fluid from pressure tank- 1 comes to the inlet of the mixer- 5 by gravity, in this case valves 2 and 12 are opened all the way; valve 23 is closed. Electric motor- 7 is started at a reduced frequency to find leakages in connection elements of the test bench. If there are no leakages, then the shaft speed is accelerated to the standard value $(50 \mathrm{~Hz})$ with the frequency converter- 6 . The fluid comes into the pump intake with the dispersing 
steps- 9 and the pump- 10; then it is sent to the gravitational separator- 13 through the discharge outlet to separate air in the test mode using the water and gas mixture. After separation, the liquid is drained into the pressure tank- 1 and the air is discharged into the atmosphere. By choke restriction of the fluid flow with the gate valve- 12 , we provide various operation modes of the researched equipment- 9, 10 with constant values of intake pressure and shaft speed. Inlet and outlet pressure of the researched pump assembly- 9,10 is measured with the pressure sensors- 4 and 11 . We measure torque on the shaft for each set operation mode with the secondary instrument of the torque coupler- 8 . In order to study the characteristics of the pumping equipment- 9,10 at another frequency, the gate valve- 12 is put back to the initial position, after which the shaft speed values of the electric motor- 7 are changed with the frequency converter- 6 . The test cycle repeats. The measurement results are entered into a table. The curves of head and power characteristics of the pumping equipment- 9, 10 for fluid medium are built after processing the parametric data.

The rheometric bench is included in the test scheme to research pumping equipment using the model gasfluid mixture. In a certain operation mode of the pump assembly- 9, 10 in fluid models, the rheometric bench is engaged by partial opening of the valve- 23 for limited supply of air to the mixer- 5. After that, the position of the gate valve- 12 is changed to change the operation modes of the pumping equipment- 9, 10. Upon completion of the tests in the set mode, the gate valve12 is returned to the initial position and the valve- 23 is slightly opened wider compared to the previous test mode. Thus, by changing the position of the valve- 23 , we can set various gas contents at the intake of the researched pumping unit- 9,10 and it is enough to provide adjustment by the gate valve- 12 to determine points of head and rate and power characteristics.

Research for determination of influence of gas bubble dispersion degree was performed in each mode to evaluate operation efficiency of the researched pump on the model Gas-Fluid Mixture (GFM). GFM samples were collected into a special chamber, which was installed at the intake of the pumping unit (at the first step) and in the section of working step No.40. GFM samples were photographed with video cameras ProgRes $\mathrm{C} 3$, with regard to preliminary enlargement of a frame using microscope Zeiss Stemi 2000-C. Later the digital pictures were processed in VideoTest-Structure 5.1 software and curves of gas bubble distribution density versus their sizes were built.

\section{Results}

According to the results of the water bench tests, in order to determine energy characteristics of dispersing steps, the following findings were obtained: Maximum efficiency factor for water- $45.4 \%$, head of one step in the optimal mode for water- $4.05 \mathrm{~m}$ and the optimal operation mode corresponds to supply of $118 \mathrm{~m}^{3} /$ day (Fig. 4).

Results of the bench tests of the pumping unit consisting of DS-100 dispersing steps and VNN-79 vortex pump on model gas-fluid mixture are presented as a graphic dependence of pressure developed by the pumping unit with supply at various volumetric rate gas contents at intake (Fig. 5). During the testing, maximum value of input gas content at the researched pump intake was recorded as $55 \%$ and the pump was operating in the non-optimal range without starvation (Fig. 5).

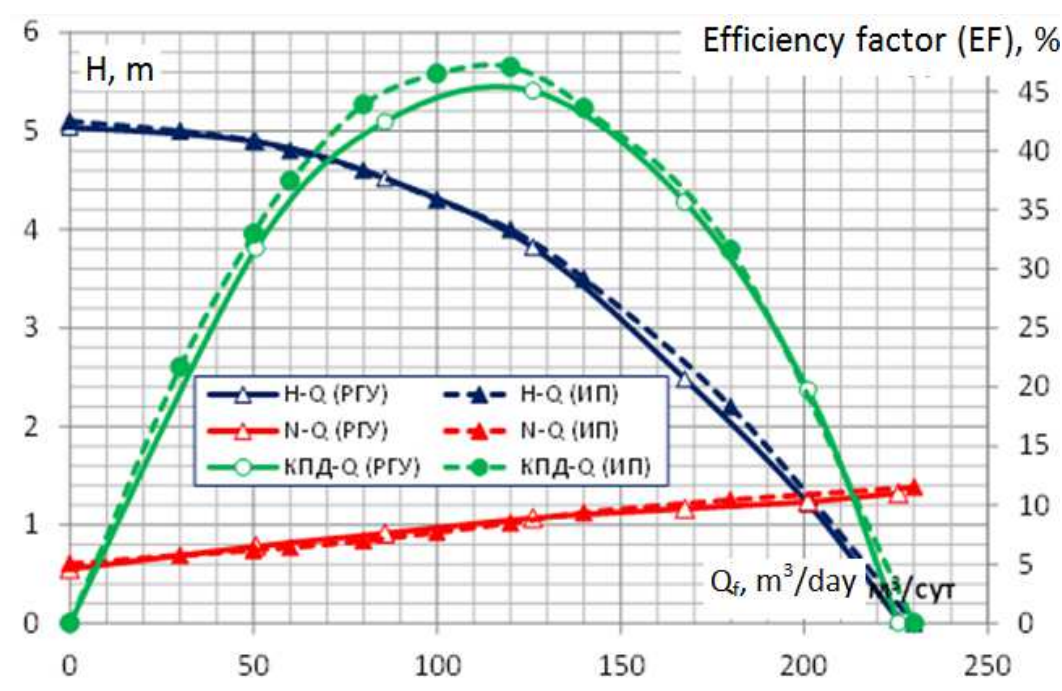

Fig. 4. Head and power characteristics of the pump with DS-100 dispersing steps ( 72 steps) at shaft speed $\mathrm{n}=50 \mathrm{~Hz}$ obtained on the bench of Gubkin Russian State University of Oil and Gas (GRSU) (solid lines) and obtained on the bench of Izhneftplast LLC (IP) (dash lines) 


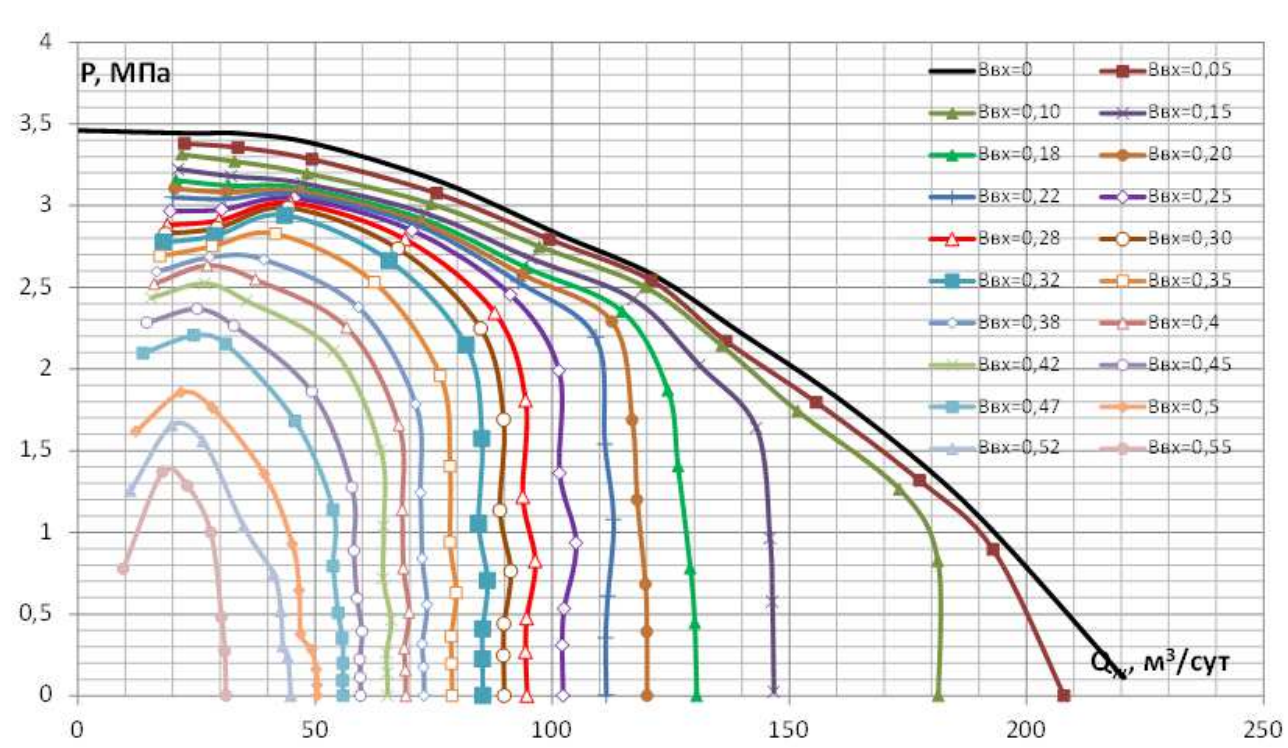

Fig. 5. Head and rate characteristics of the pumping unit consisting of DS-100 dispersing steps and VNN-79 vortex pump during suction of gas-fluid mixture "water-SAS-gas"

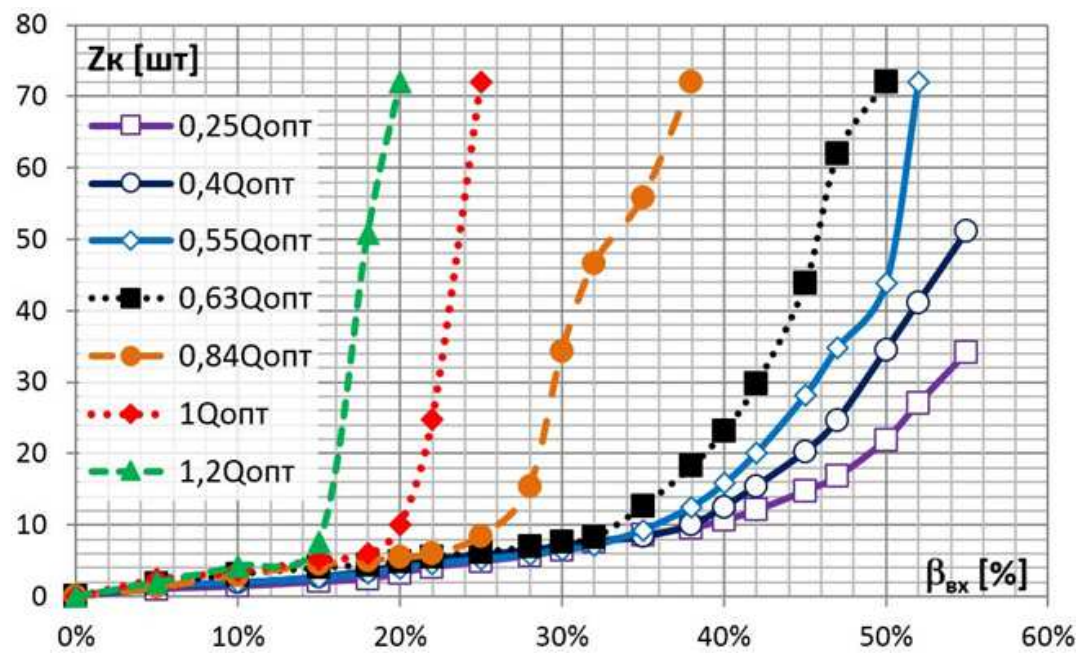

Fig. 6. Dependences of number of cavitation steps $\left(Z_{c}\right)$ of the researched pumping unit consisting of DS-100 dispersing steps and VNN-79 vortex pump from volume input gas content $B_{\text {in }}$ at various values of relative supply $Q_{o p t}$ (relativity coefficients of deliveries of the pumping unit: $0.25 ; 0.4 ; 0.55 ; 0.63 ; 0.84 ; 1.0 ; 1.2)$

Head and rate characteristics obtained during the test were converted into average integral characteristics according to the methodology (Drozdov, 1982) and quantity of cavitation steps was determined using average integral characteristics, i.e., we determined the number of steps in the pumping unit that did not develop head because of the adverse effect of free gas.

Head and rate characteristics obtained during the test were converted into average integral characteristics according to the methodology (Drozdov, 1982) and quantity of cavitation steps was determined using average integral characteristics, i.e., we determined the number of steps in the pumping unit that did not develop head because of the adverse effect of free gas.

The pump cavitation steps ensure homogenization of the gas and liquid mixture for transmission of hydraulic energy to further stages, where proper head is formed. Head created in the steps of the researched pump in some cases enables to dissolve and/or compress free gas to the size of bubbles having no adverse effect on hydraulic characteristics of researched steps of the pump.

Operating modes of the researched pump in relation to the optimal delivery parameter: $(0.25 ; 0.4 ; 0.55 ; 0.63$; 
$0.84 ; 1.0$ and 1.2$)^{*} \mathrm{Q}_{\text {opt }}$ were determined for calculation of the number of cavitation steps $\left(Z_{c}\right)$ (Fig. 6).

Pressure, gas content and density distribution curves of pumped gas-fluid mixture along the researched pumping unit were built on the basis of the obtained results. Graphs (Fig. 7) built for fluid supply at the flow rate of $76 \mathrm{~m}^{3} /$ day and with the level of input gas content of $30 \%$ are provided as an example.

Let us consider the operation mode of the researched pump with fluid supply at the flow rate of $76 \mathrm{~m}^{3} /$ day and at various values of volume input gas content (Table 1).

Results of dispersion research in two DS-100 sections showed that dispersion of gas bubbles at the intake and in the 40th step of the pump differs 3-fold (diameter of gas bubbles decreases) for the value range of volumetric input gas content: $0-50 \%$ (Fig. 8).

As volumetric input gas content increases (more than $50 \%$ ), the parameter of relative dispersion of the gas phase decreases to the value of 1.8 , which is determined by adverse effect of free gas on operation of DS-100 steps.

Parameter of relative dispersion is determined by relation of the average diameter of gas bubbles at the intake of the pumping unit to average diameter of gas bubbles in the $i$-th step (or at the pump outlet), all other conditions being equal, for example, at certain value of volumetric input gas content. If volumetric input gas content increases, the parameter of relative dispersion changes, which allows comparing various operation modes of dispersing steps of the pumping unit.

Table 1. Research results for the pumping unit consisting of DS-100 dispersing steps and VNN-79 vortex pump in the mode: $\mathrm{Q}_{\mathrm{fl}}=76$ $\mathrm{m}^{3} /$ day with ensured collection of samples at the pumping unit intake and in the section of DS-100 dispersing step No. 40 (count of step number begins from the pumping unit intake)

\begin{tabular}{|c|c|c|c|}
\hline $\begin{array}{l}\text { No. } \\
\text { Sample number }\end{array}$ & $\begin{array}{l}B_{\text {in }}, \% \\
\text { Volume input gas content }\end{array}$ & $\begin{array}{l}\mathrm{D}_{\text {aver.in }} \text {, mcm } \\
\text { Mean diameter of gas bubbles in GFM } \\
\text { flow at the intake of the pumping unit }\end{array}$ & $\begin{array}{l}\mathrm{D}_{\text {aver.40 }}, \mathrm{mcm} \\
\text { Mean diameter of gas bubbles in } \\
\text { GFM flow at dispersing step No.40 }\end{array}$ \\
\hline 1 & 24 & 108 & 36 \\
\hline 2 & 32 & 136 & 46 \\
\hline 3 & 49 & 143 & 47 \\
\hline 4 & 51 & 175 & 56 \\
\hline 5 & 52 & 167 & 70 \\
\hline 6 & 53 & 172 & 95 \\
\hline
\end{tabular}

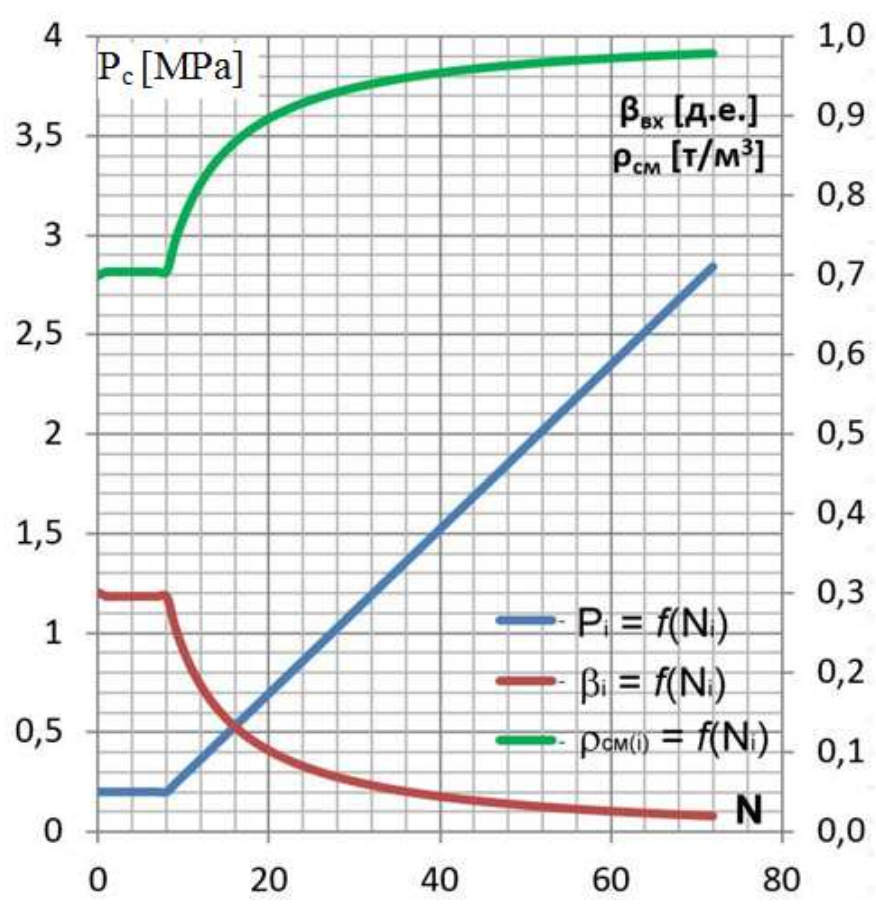

Fig. 7. Distribution of pressure, gas content and density of GFM along the length of the pumping unit consisting of DS-100 dispersing steps and VNN-79 vortex pump tested on model mixture "water-SAS-gas" at $\mathrm{Q}_{1}=76 \mathrm{~m}^{3} /$ day and at volume input gas content $B_{\text {in }}=30 \%$ 


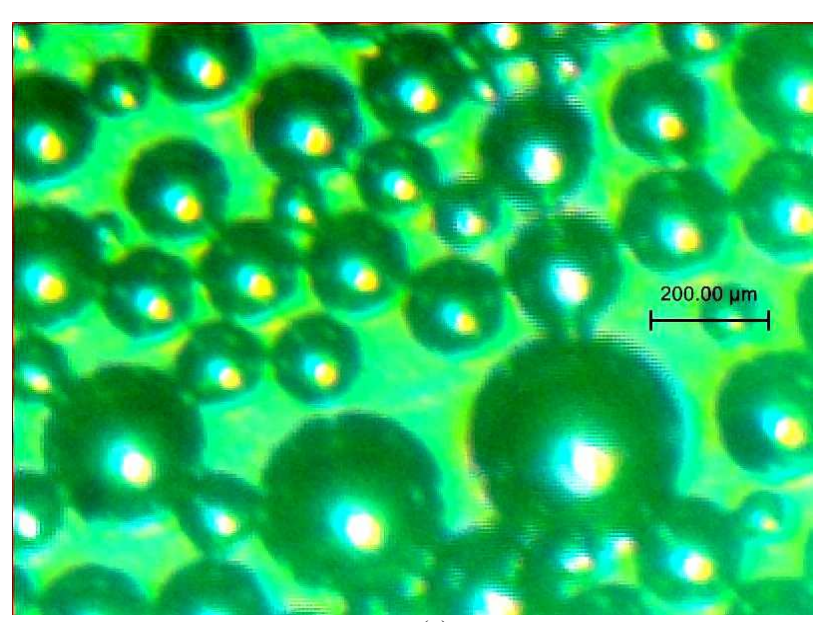

(a)

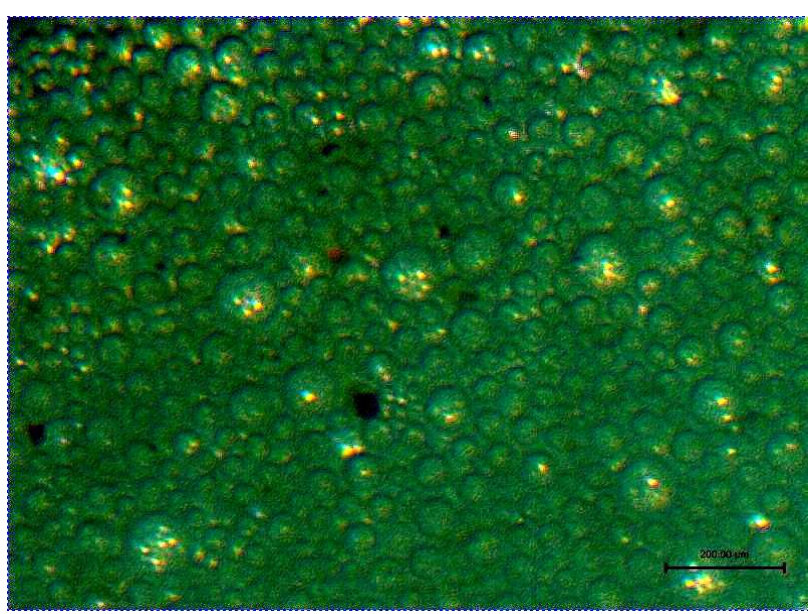

(b)

Fig. 8. Fragment of pictures with samples of the gas-fluid mixture at the intake (a) and in the $40^{\text {th }}$ step (b) of the pumping unit (DS100 dispersing steps) in accordance with the operation mode of the pumping unit: $\mathrm{P}_{\mathrm{in}}=0.2 \mathrm{MPa}, \mathrm{n}=50 \mathrm{~Hz}, \mathrm{Q}_{\mathrm{fl}}=48 \mathrm{~m}^{3} / \mathrm{day}$ and $B_{\text {in }}=40 \%$

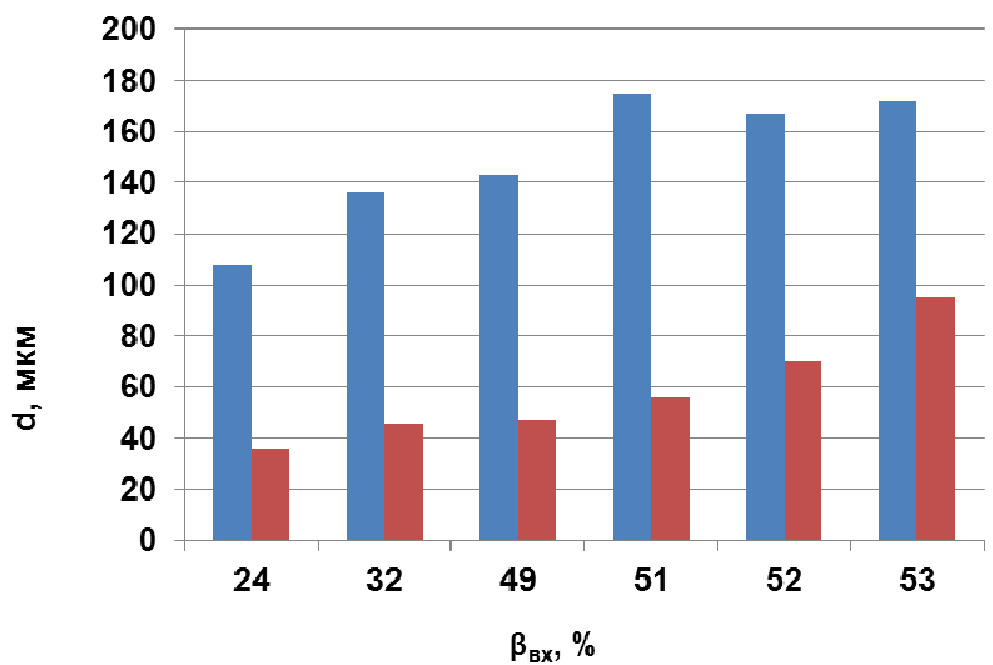

шcp.вx, мкм

च dcp.40, мкм

Fig. 9. Dependence of critical diameter of gas bubbles in GFM flow on volumetric input gas content at the intake of the researched DS-100 dispersing stages

Therefore, based on the results of the bench test, technical range of effective use of DS-100 dispersing steps can be determined by the parameter of volume input gas content: $0-50 \%$ (Fig. 9).

\section{Discussion}

Based on the results of the carried out research, graphic dependencies were built to determine operation efficiency of the pumping unit with DS-100 dispersing steps (Fig. 10). The presented dependencies allow determining the required quantity of dispersing steps within the pumping unit at various volumetric values of residual and input gas content. Assume that it is necessary to select a submersible pumping unit for work in conditions of volumetric input gas content of $40 \%$ for operation of a well with fluid discharge of 76 $\mathrm{m}^{3} /$ day, then the submersible pumping unit should consist of DS-100 dispersing steps and classic ETsN-80 steps or steps with a vortex crown of VNN-79 type. 


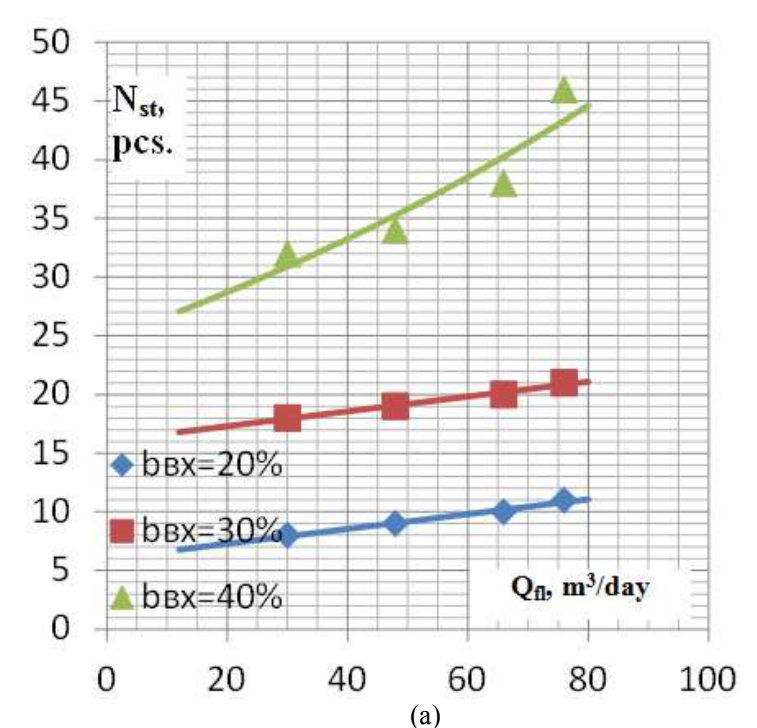

(a)

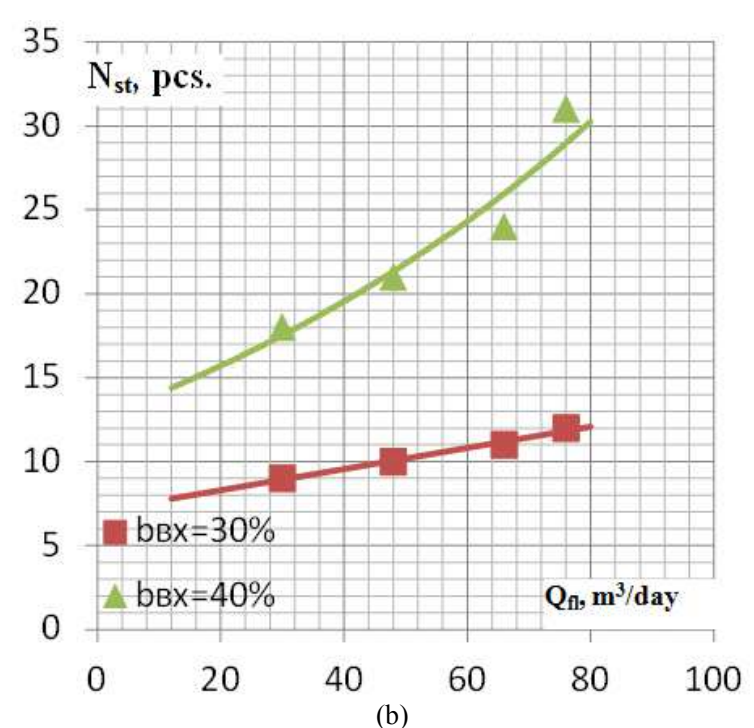

(b)

Fig. 10. Graphic dependencies for determination of required minimal quantity of DS-100 dispersing steps within the pumping unit: DS-100-VNN-79 to ensure stable operation with residual gas content: $10 \%$ (a) and $20 \%$ (b) and at volumetric input gas content values: 20,30 and $40 \%$

Using the obtained dependencies, we determine the required quantity of dispersing steps for the pumping unit: ETsN-80- DS-100 (Fig. 10a). To do this, we determine the value on the supply axis- $76 \mathrm{~m}^{3} /$ day, then draw a straight line in parallel to the step quantity axis till the intersection with the curve in the thought for volumetric input gas content value- $40 \%$, then from the intersection point we draw a line till the intersection with the step quantity axis in the thought and get the number of dispersing steps- 42 .

\section{Conclusion and Further Research}

Thus, the results of bench research of DS-100 dispersing steps as a part of the pumping unit on the model gas-fluid mixture allow drawing the following conclusions:

- A method for estimation of quantity of dispersing steps during operation of the pumping unit on gasfluid mixture under various conditions was developed in order to determine efficiency of gas dispersion in the pumping unit with DS-100 dispersing steps

- The bench research of characteristics of DS-100 dispersing steps allowed determining the application range by the parameter of volumetric input gas content: $0-50 \%$

- Graphic dependencies were developed enabling to determine rational quantity of dispersing steps within the submersible pumping unit

- The obtained results of the research are recommended for industrial application when selecting submersible electrical centrifugal pumping units for wells with high gas factor

\section{Acknowledgement}

The authors would like to express their gratitude to the management of the Gubkin Russian State University of Oil and Gas for their support in conducting this research.

\section{Funding Information}

Scientific and research works on improvement and creation of new methods for energy-efficient protection of ECP units from adverse effect of free gas are carried out at Gubkin Russian State University of Oil and Gas within the framework of implementation of federal target program "Research and Developments in Priority Directions of Scientific and Technological Complex of Russia for 2014-2020" with the financial support from the Ministry of Education and Science of the RF (unique identifier RFMEFI57714X0126) and with support from the industrial partner Izhnefteplast LLC.

\section{Author's Contributions}

Vladimir Verbitsky: Organized the study, information checking and manuscript writing.

Aleksey Dengayev: Engaged in the results analysis, drafting of article.

Oleg Zubkov: Responsible for data collecting and analysis of the results. 
Dmitry Koshkin: Contributed in data collecting, writing and editing of the manuscript.

\section{Ethics}

The authors have no conflicts of interest in the development of the research and publication of this article.

\section{References}

Dengaev, A.V., 2005. Efficiency increase of well operation with submersible centrifugal pumps during suction of gas-fluid mixtures. $\mathrm{PhD}$ Thesis, Moscow.

Dengayev, A.V., A.N. Drozdov and V.S. Verbitsky, 2005. Investigation of causes of "falls" of gas separators within ECP unit. Oil and Gas Territory, 11: 50-54.
Drozdov, A.N., 1982. Development of a method for computation characteristics of submersible centrifugal pump during operation of wells at low pressures at pump inlet. PHD Thesis, Moscow.

Drozdov, A.N., V.S. Verbitsky, D.N. Lambin and A.V. Dengaev, 2011. Stand research and analysis of average-integral characteristics of submersible centrifugal pumps operating at gas-liquid mixtures. Proceedings of the SPE Production and Operations Symposium, Mar. 27-29, SPE, Oklahoma City. DOI: $10.2118 / 141291-M S$

Statistics, 2015. Oil and gas vertical.

Vasiliev, Y.N., R.A. Maksutov and A.I. Bashkirov, 1961. Experimental study of oil and gas structure in a flowing well. Oil Industry, 4: 41-44. 